\title{
Marianne sur les planches : les héroïnes anonymes du théâtre de la Révolution française (1793-1798)
}

Marianne on the Stage: Anonymous Heroines in the Theater of the French Revolution (1793-1798)

\section{Eva Bellot}

\section{OpenEdition}

Journals

\section{Édition électronique}

URL : https://journals.openedition.org/ahrf/12422

DOI : $10.4000 / a h r f .12422$

ISSN : 1952-403X

Éditeur :

Armand Colin, Société des études robespierristes

Édition imprimée

Date de publication : 1 mars 2012

Pagination : 69-92

ISSN : 0003-4436

Référence électronique

Eva Bellot, « Marianne sur les planches : les héroïnes anonymes du théâtre de la Révolution française (1793-1798) », Annales historiques de la Révolution française [En ligne], 367 | janvier-mars 2012, mis en ligne le 28 septembre 2012, consulté le 01 juillet 2021. URL : http://journals.openedition.org/ahrf/ 12422 ; DOI : https://doi.org/10.4000/ahrf.12422 


\title{
MARIANNE SUR LES PLANCHES : LES HÉROÏNES ANONYMES DU THÉÂTRE DE LA RÉVOLUTION FRANÇAISE (1793-1798)
}

Eva BELLOT

\begin{abstract}
Les faits historiques du théâtre de la Révolution française (1789-1799) mettent en scène une promotion de la figure de l'héroïne populaire. À travers le poids dramatique et idéologique qu'elles acquièrent, ces figures féminines passent du statut d'anonymes à celui d'héroïnes mythiques et emblématiques de la lutte républicaine. Au-delà des stéréotypes sur l'exclusion des femmes du phénomène révolutionnaire, ces pièces construisent une représentation contrastée, permettant de réévaluer la place symbolique et sociale des femmes en Révolution.
\end{abstract}

Mots-clés : Héroïne, anonyme, Révolution, trait historique, allégorie, démocratisation

Lélection de Marianne comme allégorie de la République ne constitue pas l'un des moindres paradoxes de la Révolution. Tour à tour qualifiée de " gueuse » ou d'idole, tantôt mère de la patrie ou vierge républicaine, sa légitimité emblématique a été sujette à de nombreux débats dès 1792, qui remettaient en cause le choix de l'allégorie féminine pour symboliser la République ${ }^{1}$. Relent de misogynie de la part des députés

(1) Sur ce point voir Lynn Hunt, « Pourquoi la République est-elle une femme? La symbolique républicaine et l'opposition des genres » dans Michel Vovelle (dir.), Révolution et République. L'exception française, Actes du colloque de Paris I - Sorbonne, 21-26 septembre 1992, Paris, Éditions Kimé, p. 358-365; voir aussi Madelyn Gutwirth, «The Rights and Wrongs of Woman : The Defeat of Feminist Rhetoric by Revolutionary Allegory », L'Encyclopédie, Diderot, l'esthétique : Mélanges en hommage à Jacques Chouillet (1915-1990), Paris, PUF, 1991, p. 150-68. 
de la Convention? C'est en tout cas la représentation qui a longtemps prédominé dans l'imaginaire collectif : les femmes, reléguées au rang de parentes pauvres de la Révolution, reconnues citoyennes sans pouvoir exercer pleinement leurs droits civiques, auraient été les grandes absentes du processus révolutionnaire. Certaines études d'historiens des idées ont néanmoins permis de réévaluer partiellement ce préjugé, en montrant que l'exclusion politique de fait n'avait pas empêché une influence au niveau des représentations et de la mentalité contemporaines ${ }^{2}$. La cocarde arborée par Marianne n'était pas exclusivement ornementale.

Si Marianne constitue un point de départ intéressant pour aborder la question des femmes, c'est parce qu'elle invite d'emblée à penser la question de la sexualisation des représentations allégoriques qui ont structuré la mentalité révolutionnaire tant sur un plan politique concret que dans les schémas populaires inconscients ${ }^{3}$. Une fois le meurtre de la mère-reine accompli, la génération des filles de la Révolution a donné lieu à bien des fantasmes idéologiques prenant souvent la forme de schémas antagonistes : féminité ou androgynie, maternité ou virginité républicaine, famille ou patrie. Au même titre que leurs homologues masculins, les femmes de la Révolution n'ont pas échappé au processus d'héroïsation massive encouragé par les autorités. La Convention nationale décréta un vaste élan de régénération morale ${ }^{4}$, auquel les artistes furent associés : lors du célèbre concours de peinture de l'an II, de nouveaux héros populaires firent leur apparition parmi les sujets représentés, empruntés pour la plupart au Recueil des actions hérö̈ques et civiques des Républicains ${ }^{5}$ diffusé par les autorités. Ces nouveaux exemples de héros citoyens accompagnèrent une redéfinition élargie de la notion d'héroïsme, ne s'apparentant plus seulement à l'exploit militaire mais intégrant la repré-

(2) Voir sur cette question les ouvrages suivants : Paule-Marie DuHET, Les Femmes et la Révolution, 1789-1794, Paris, Gallimard, 1971; Dominique Godineau, Citoyennes tricoteuses. Les femmes du peuple à Paris pendant la Révolution française, Aix-en-Provence, Alinéa, 1989; Jacques Guilhaumou et Martine LAPIED, "L'action politique des femmes pendant la Révolution française », dans Christine Fauré (dir.), Encyclopédie politique et historique des femmes, Paris, PUF, 1997, p. 139-168.

(3) Voir notamment Lynn Hunt, Le Roman familial de la Révolution française, traduction Jean-François Sené, Paris, Albin Michel 1995 (titre original : The Family Romance of the French Revolution, Berkeley, 1992, University of California Press). L'historienne transpose la grille d'interprétation freudienne sur 1'histoire révolutionnaire et propose une représentation archétypale du fait social par le biais de la fantasmagorie familiale.

(4) Serge Bianchi, La Révolution culturelle de l'an II, élites et peuples, Paris, Aubier, 1982.

(5) Bourdon de la Crosnière et Thibaudeau, Recueil des actions héroïques et civiques des Républicains français, Paris, Imprimerie Nationale, an II. 
sentation d' « actions vertueuses » telles que le sacrifice désintéressé ou la piété filiale. La mutation héroïque investissait désormais la sphère domestique en glorifiant les héros du quotidien au-delà des hiérarchies sociales, générationnelles et sexuelles. Ce processus de démocratisation héroïque n'épargna aucun pan de la société révolutionnaire, qu'il concerne les grands hommes de la Révolution - Marat, Le Peletier, Desilles - ou de jeunes citoyens issus du peuple - Agricol Viala ou le jeune Barra - s'étant distingués par une action républicaine. L'élargissement pyramidal de l'accès à la dignité héroïque concerna aussi les femmes, citoyennes anonymes dont - et c'est là un phénomène symptomatique - les noms ont été oubliés pour la plupart, victimes de l'euphorie exaltée pour ces gloires de l'instant qui ne parvinrent pas à survivre dans la mémoire collective au-delà de la décennie révolutionnaire. Deux exemples, tirés eux aussi du Recueil des actions hérö̈ques et civiques des Républicains français, se singularisent parmi la galerie des citoyennes anonymes populaires : l'héroïne de Saint-Milhier et la citoyenne Bergougnoux. Excepté ces deux figures - dont il faut d'ailleurs relativiser la popularité, très circonscrite à notre période - l'héroïne révolutionnaire est retombée dans l'anonymat aussi rapidement qu'elle en était sortie.

Si l'iconographie et les recueils d'éducation furent les principales sources de ce répertoire héroïque féminin, le théâtre servit aussi d'instrument de la popularisation de ces héroïnes républicaines. À défaut d'accès à l'égalité civile et politique, le personnage féminin conquit l'égalité dramatique sur les planches. Une étude sur l'héroïne dans le théâtre de la Révolution nécessiterait qu'on y consacre un volume entier, raison pour laquelle nous avons choisi de restreindre notre approche à une partie du répertoire souvent délaissée, à savoir la catégorie des faits historiques en un ou deux actes mettant en scène une héroïne historique ou présentée comme telle 6 . Ces petites pièces, souvent données à l'issue d'une grande pièce en cinq actes, constituent un abondant corpus à la fois cohérent dans son ambition et hétéroclite dans ses réalisations. Cohérent parce que le sujet de leur intrigue et la représentation de cette dernière s'apparentent à une commémoration républicaine tendant à l'héroïsation frénétique en masse. Hétéroclite aussi car les représentations héroïques n'ont cessé de

(6) Nous incluons dans cet ensemble toutes les formes brèves qui ne présentent pas nécessairement la qualification générique de « fait historique » ou « trait historique » mais qui s'apparentent à ce sous-ensemble par la représentation d'une action simple, centrée sur la question de l'exploit ou l'acte de bravoure d'une héroïne anonyme, et qui se présentent comme la transposition dramatique d'une anecdote historique. 
se fissurer et de se déplacer au cours de la décennie où la rupture politique marquée par Thermidor amorce un infléchissement des représentations de l'hérö̈ne. Nous avons ainsi sélectionné une dizaine de faits historiques ${ }^{7}$, tous écrits et représentés entre 1793 et 1798, qui témoignent des mutations de la représentation de l'héroïne, entre conformisme républicain et résistance idéologique.

La plupart des travaux sur la question de l'héroïne ont été entrepris par des historiens de l'art ou des idées, notamment à travers des travaux sur l'iconographie et l'histoire de la société ${ }^{8}$. Quelques héroïnes célèbres de l'Histoire comme Olympe de Gouges ou Théroigne de Méricourt ont, quant à elles, fait l'objet de biographies et de monographies ponctuelles'. La question de l'héroïne dramatique - en dehors du cas Charlotte Corday ${ }^{10}-$ n'a pas suscité d'étude synthétique ni même partielle. Or la démocratisation héroïque amorcée sous l'ère révolutionnaire invite à réévaluer l'impact dramatique des personnages féminins. Au moment où la République élit ses figures tutélaires, la question de la place dévolue à l'héroïne dans le répertoire apparaît légitime et peut sans doute contribuer à enrichir l'histoire des représentations. La mise en application dramatique des actions héroïques rapportées dans les recueils à l'usage du peuple participe du processus d'héroïsation de la citoyenne anonyme par la médiation du théâtre. À la conception traditionnelle de l'héroïne tra-

(7) Nous les énumérons par ordre chronologique de première représentation, entre 1793 et 1798 : Citoyenne VILler, Barra ou la mère républicaine; Plancher DE VAlCour, La Discipline républicaine; J.B. Radet, Le Canonnier convalescent; Citoyen Philipon, Agricol Viala ou le jeune héros de la Durance; Citoyen LAUs, La Vraie républicaine ou la voix de la patrie; VeE et BARRAL, L'hérö̈ne de Mithier; Citoyens VILLER et Gouffe, Cange ou le commissionnaire bienfaisant; F.M. Riou et J. Pain, Les Chouans ou la républicaine de Malestroit; SEgur Le Jeune, Élize dans les bois; J. N. BoulLLy, Léonore ou l'amour conjugal.

(8) Voir notamment les travaux de Lynn Hunt, Politics, culture and class in the French Revolution, Berkeley, University of California Press, 1984. Voir également Michel VovelLe, La Mentalité révolutionnaire, société et mentalités sous la Révolution française, Paris, Éditions sociales, 1985 et Les Images de la Révolution française, Paris, Publications de la Sorbonne, 1988; voir aussi Maurice Agulhon, Marianne au combat : l'imagerie et la symbolique républicaines de 1789 à 1880, Paris, Flammarion, 1979.

(9) Olivier Blanc, Marie-Olympe de Gouges, une humaniste à la fin du XVIII ${ }^{e}$ siècle, Paris, Éditions René Viénet, 2003; Élisabeth Roudinesco, Théroigne de Méricourt, une femme mélancolique sous la Révolution, Paris, Seuil, 1989, rééd. Albin Michel, 2010.

(10) Encore que l'abondance des travaux critiques sur Charlotte Corday concerne surtout la question de son rôle et de sa réception historique. Les représentations dramatiques et esthétiques de l'héroïne n'ont pas donné lieu à une synthèse globale. Voir La Mort de Marat, Jean-Claude BonneT (dir.), Paris, Flammarion, 1986; Comment en finir avec la Révolution : l'apothéose de Charlotte Corday et d'Élisabeth de France dans le théâtre de Thermidor, Société française d'Étude du XVIII $^{\mathrm{e}}$ siècle, Publications de l'Université de Saint-Étienne, 1998; Guillaume MAZEAU, Le bain de l'histoire : Charlotte Corday et l'attentat contre Marat (1793-2009), Seyssel, Champ-Vallon, 2009. 
gique tirée de l'Antiquité gréco-romaine ou des modèles aristocratiques de l'Ancien Régime se substitue la figure de l'héroïne républicaine au cœur de l'action dramatique et du projet idéologique qui la motive. «On peut être héros sans ravager la terre ${ }^{11}$ », écrivait Boileau dans ses Épitres, sanctionnant la dichotomie entre l'héroïsme homérique des exploits militaires et l'héroïsme de caractère, celui de la grandeur d'âme et de l'exercice de la vertu. La place dévolue à l'héroïne au sein de ce répertoire à l'usage des classes populaires semble accréditer cette représentation d'un héroïsme intériorisé et spontané. Non qu'il faille lire dans ce corpus les signes anachroniques d'une revendication féministe; néanmoins, cette promotion héroïque révèle que quelque chose d'ordre politique se construit dans la mise en place de l'ethos stéréotypé du personnage féminin. Ces figures, dont la plupart sont présentées comme des anecdotes historiques réelles, participent d'une volonté de mythification du corps populaire par la vertu accessible de l'exemplarité. Elles esquissent tour à tour les multiples visages d'une Marianne personnifiée en chair et en os, bien avant que le buste de marbre ne vienne définitivement figer l'allégorie républicaine.

\section{L'héroïsme du quotidien : mise en application dramatique}

Sans doute faut-il au préalable évoquer ce qui constitua le support principal des auteurs dramatiques pour élaborer leur intrigue et le personnage de l'héroïne républicaine. La presse et les recueils héroïques diffusés par la Convention fournirent les principaux sujets par l'abondance et la diversité inédite des modèles héroïques qu'ils proposaient. Dans le préambule du Recueil des actions hérö̈ques et civiques des Républicains français, les auteurs justifient le critère idéologique qui a présidé à la sélection des anecdotes :

« Nous aurions pu remplir ce numéro et beaucoup d'autres ensuite, de récits plus saillants; nous aurions pu y réunir un ensemble de traits tous plus héroïques les uns que les autres; de ces traits qui provoquent d'autant plus l'admiration qu'ils paraissent surpasser les forces ordinaires de la nature. (L'énergie des Républicains français, le sublime enthousiasme de la Liberté, qui élève l'homme au-dessus de lui-même, nous garantissait que les matériaux ne nous manqueraient pas). Mais nous avons voulu ménager les jouissances de nos lecteurs : nous avons pensé que des traits

(11) Nicolas Boileau, Art poétique, Epître I, « Au Roi », v. 94. 
de probité, de désintéressement dont la Convention nationale avait entendu le récit avec intérêt, figureraient sans désavantage à côté de traits d'héroïsme, dans un recueil dont l'objet est de présenter à la jeunesse française le tableau des vertus de ses pères et de ses contemporains, d'exciter et d'entretenir la sensibilité si naturelle à cet âge.

Voici la marche que nous avons suivie. Chaque numéro contiendra d'abord un récit des premiers événements de la révolution; les différents traits d'héroïsme et de civisme seront variés de manière à éviter l'uniformité : tantôt, ce sera un trait de désintéressement; une action héroïque lui succédera et sera suivie d'un sentiment de piété filiale $\gg^{12}$.

Par l'introduction de ce texte justificatif du projet idéologique à l'origine de la rédaction du recueil, les députés de la Convention reconnaissent la diversité des actions héroïques à travers un spectre élargi. Dans une volonté pédagogique manifeste, ils veulent ancrer chez les futurs citoyens l'idée que la valeur n'attend pas le nombre des années et que tout acte de dévouement peut s'avérer profitable à la République. La sélection opérée par le recueil laisse donc place à un large éventail de représentations par l'évocation de héros d'âge et de sexe différents. En promouvant les formes d'un héroïsme domestique du côté de la vertu plus que de l'exploit, la nouvelle Bible républicaine prend garde de n'exclure aucun citoyen. La démarche consiste par ailleurs à relater le fait le plus simplement et le plus brièvement possible, sans effet d'emphase ni de style recherché. D'où l'aridité narrative de ces récits héroïques provinciaux accumulés en catalogue.

La mise en application dramatique des ces faits héroïques correspondait à une double ambition : sur un plan idéologique, il s'agissait d'étendre la portée de ces anecdotes historiques provinciales au niveau national au même titre que les grands hommes de la Révolution étaient devenus l'objet d'un culte populaire; sur le plan dramaturgique, la représentation in medias res de l'héroïne citoyenne faisait appel à une dynamique de catharsis positive fondée sur la vertu de l'exemple. Avec l'avènement de l'héroïne anonyme mythifiée, la mobilisation républicaine gagne la sphère des théâtres de province. Grâce à la promotion de cette figure populaire, les hommes de l'an II espéraient éveiller et toucher la fibre républicaine inscrite dans les âmes citoyennes. D'autant que le courant idéologique traditionnel associant la féminité aux vertus de dévouement et de sacrifice,

(12) Bourdon de la Crosnière et Thibaudeau, op. cit., p. 3-4. 
et donc à l'espace domestique plutôt qu'à la sphère publique, faisait partie intégrante des représentations entretenues par les autorités jacobines à partir de l'an II. Dans un article consacré à la figure de la femme révolutionnaire au théâtre entre 1789 et 1794, Annette Graczyk écrit :

« D’une manière générale, elles [les femmes] sont solidement arrimées à une conception de la nature éminemment rousseauiste. Au fond, c'est l'assimilation de la femme à la nature -une nature telle que la voit la philosophie des Lumières - qui en fait cet excellent élément de la symbolique révolutionnaire et lui confère ses propriétés allégoriques de la déesse de la Liberté. Pendant la domination jacobine la féminité devient, sur scène, accessoire d'un paysage bucolique. Elle symbolise l'état d'innocence naturelle que la Révolution veut réinstaurer dans toute son évidence première. À l'instar du bon sauvage - non moins fictif - la « nature » féminine prend les traits d'une nature domestiquée dont les aspects menaçants sont jugulés par l'assignation à son rôle social d'épouse et de mère. Quand on attend des femmes l'engagement civique et le patriotisme, on ne les sollicite que dans le cadre restreint de leur féminité : la femme incarne et contrôle l'aspect émotionnel de la Révolution. Elle sert d'instance régulatoire en influençant le civisme de son mari et de son fils. La justesse sociale de son instinct exclut les mariages politiquement douteux, la fait démasquer les contre-révolutionnaires et les opportunistes $\gg^{13}$.

Ce point de vue souligne deux aspects essentiels de la représentation féminine dans le répertoire : d'un côté l'ancrage de la femme dans un rôle familial - épouse ou mère - par la référence à une forme d'instinct biologique de préservation et d'innocence morale, de l'autre le rôle politique d'instance régulatrice qui la condamne peu ou prou à l'inaction ou bien à l'action de second plan.

Or cette représentation ne recouvre que partiellement la réalité dramatique des textes : s'il est vrai que toutes les héroïnes de notre corpus sont définies comme épouses et mères, elles n'en sont pas moins au cœur de l'action dramatique puisqu'elles agissent et prennent l'initiative du mouvement de sursaut républicain. La forme du fait historique se prêtait particulièrement bien à la représentation d'une action simple et exemplaire destinée à animer les vertus républicaines et l'esprit de patriotisme. Il n'est pas rare que la représentation mêle à la fois la représentation du

(13) Annette GraczyK, «L'image de la femme révolutionnaire dans le théâtre français de 1789 à 1794 », Studies on Voltaire and the Eighteenth Century, n 265, 1989, p. 1327. 
trait héroïque et sa célébration immédiate sous forme d'un vaudeville ou d'un chœur final dont la portée encomiastique s'accompagne d'une intention pédagogique. Dans la scène finale du Canonnier convalescent ${ }^{14}$, fait historique de l'an II, la jeune Julie, qui a sauvé et soigné au péril de sa vie un canonnier républicain, du nom de Beltonis, blessé lors d'un combat, se voit remettre une couronne civique par le maire du village devant les citoyens assemblés. Le commissaire prononce alors un fervent éloge de ce dévouement républicain dont il est inutile de souligner la portée métathéâtrale :

«Citoyens, c'est par un semblable dévouement qu'on acquiert et qu'on maintient la Liberté; prenez Beltonis pour modèle et vous serez dignes du beau titre de Républicain. Et vous, citoyennes, imitez l'intéressante Julie; soyez sensibles et bienfaisantes, comme elle, envers tous nos braves frères d'armes, et vous aurez, comme elle, bien mérité de la patrie $»^{15}$.

Et de conclure quelques lignes plus loin dans le vaudeville sur l'exemplarité morale de la conduite de Julie pour la jeune génération de citoyens : "Ah! dès leur plus tendre jeunesse, / De nos enfants formons le cœur; / A leurs yeux pratiquons sans cesse / Les vertus qui font le bonheur. / Avant que des écrits civiques / Puissent guider leurs jeunes ans, / Que le tableau des mœurs publiques / Soient le livre de nos enfants $»^{16}$. L'assimilation du fait historique à un tableau dramatique vivant des vertus républicaines inscrit en creux la vocation pédagogique à l'œuvre.

L'apothéose scénique de ces gloires anonymes éphémères repose sur la dramatisation d'une seule action qui vise à souligner la constitution d'un ethos héroïque dans une dynamique de révélation. Ces héroïnes anonymes issues du peuple ont su se distinguer par un acte hors-norme le plus souvent présenté comme un acte de révolte ou de résistance politique face à un péril républicain. Leur héroïsme n'excède pas l'instant de leur geste, après quoi elles s'en retournent à une vie laborieuse. D'autant qu'à la différence d'un Barra ou d'un Agricol Viala, les hérö̈nes anonymes n'accèdent pas à la dignité suprême du martyr tragique : elles ne meurent pas et ne se suicident pas malgré leur sens aigu du sacrifice. Leur bra-

(14) Jean-Baptiste RADET, Le Canonnier convalescent, fait historique en un acte et en vaudevilles, représenté pour la première fois sur le Théâtre du Vaudeville le 11 messidor an II (29 juin 1794), Paris, chez le Libraire, au Théâtre du vaudeville, an II.

(15) Jean-Baptiste RADET, ibid., scène 13, p. 42.

(16) Ibid., p. 42. 
voure est entièrement concentrée dans l'action républicaine, soulignant le dévouement sans faille à la patrie. L'exemple le plus célèbre parmi notre corpus est représenté par un fait historique en un acte des citoyens Vée et Barral, intitulé L'Hérö̈ne de Mithier ${ }^{17}$, qui fut représenté en l'an II et s'inscrit en droite ligne dans l'idéologie jacobine. L'héroïne de Mithier connut une popularité rapide grâce à la propagande robespierriste qui récupéra ce fait divers hors-norme pour l'ériger en mythe républicain : l'épisode suscita notamment une vogue dans l'iconographie contemporaine ${ }^{18}$. Dans le domaine théâtral en revanche, le fait historique des citoyens Vée et Barral constitue la seule pièce imprimée qui nous soit parvenue.

L'héroïne éponyme, prénommée Catherine et femme du forgeron Sans Quartier, est présentée comme une figure de la résistance dans le combat qui oppose les Républicains aux Vendéens insurgés. Pétrie d'idéalisme républicain, Catherine élève ses enfants dans le culte des valeurs jacobines. Dans la scène 6 , tandis que les personnages sont répartis de sorte à former un tableau familial républicain, elle fait lire à son jeune fils, Sans Souci, les tables de la loi républicaine qu'elle relaie avec ferveur - il s'agit ici d'un passage chanté - : «Sans Souci, lisant : Vois com' un frère / Chaqu' citoyen / Catherine, vivement : Ô, mon fils ! retiens bien / Cette maxime chère (bis) / Soutiens tes droits, / Respect aux lois; / Brûle d'amour pour la patrie $\rangle^{19}$. Lorsque le tocsin sonne, signe de l'attaque prochaine des Vendéens et d'une menace de combat imminente, Catherine, loin de se morfondre dans une douleur pathétique, prononce un beau morceau d'éloquence républicaine teinté de sublime :

«TYRANS! Voilà le peuple que vous osez combattre! Depuis l'enfant sortant à peine du berceau, jusqu'au vieillard près de sa tombe, tout est électrisé du même feu, du feu sacré de la Liberté! et vous croyez nous réduire ?... Non!... non!... non! jamais. La Liberté ou la mort !... (Après un moment de réflexion) Que les ennemis, s'ils viennent jusqu'ici, m'a dit Sans Quartier, y trouvent leurs tombeaux, et nous des vengeurs... Oui, je

(17) Note de la rédaction : l'orthographe de cette héroïne varie selon les productions. VÉE et BARRAL, L'Héroïne de Mithier, fait historique en un acte et en prose, représenté pour la première fois sur le Théâtre du Vaudeville, le 3 fructidor an II (20 août 1794), Paris, chez le Libraire, au Théâtre du Vaudeville, an II.

(18) La plus célèbre est la gouache de Jean-Baptiste LesuEur, intitulée L'Hérö̈ne de Milhier et accompagnée d'une légende résumant l'anecdote, 1793, Paris, Musée Carnavalet. Sur le mythe de l'héroïne de Mithier (dont l'orthographe du nom a subi quelques variations) et ses transformations, voir Claude Langlois, «Les dérives vendéennes de l'imaginaire révolutionnaire », Annales. Économies, sociétés, civilisations, 1988, volume 42, nº 3, p. 771-797.

(19) VÉE et BARRAL, op. cit., scène 6, p. 19. 
serai digne de toi, et tes fils suivront ton exemple; si je leur donnai des leçons de civisme, de courage, c'est le moment d'en profiter; la Liberté enfanta des héros, je veux que mon sexe en compte aussi; d'ailleurs, quand le danger est commun, tout le monde doit combattre $»^{20}$.

L'héroïne se transforme dans ce passage en allégorie personnifiée de la République, reprenant à son compte la devise républicaine exclusive des Jacobins de l'an II. Son discours conteste également l'exclusion des femmes de la lutte armée sous prétexte d'une exception sociale qui les cantonnerait à la sphère du foyer : dès lors que la République est en danger, toute âme citoyenne, quel que soit son sexe, doit s'engager ardemment dans la résistance. Le geste héroïque de l'héroïne est ainsi préparé en amont de l'intrigue par la peinture d'un caractère exceptionnel. Lorsque les Vendéens pénètrent le seuil de sa maison, Catherine s'empare d'un pistolet et vise un baril de poudre (précédemment apporté par son mari en prévision des combats), moment d'acmé dramatique intense :

«UN BRIGAND, s'avançant davantage : Non, point de grâce.

CATHERINE, avec force: Point de grâce!... Non point de grâce. (Elle jette la couverture du baril à bas, leur présente un pistolet, et l'autre sur la poudre.) Brigands, au nom de la république, armes bas, ou vous sautez.

Les brigands regardent du côté de la porte.

CATHERINE : Ne reculez, ni n'avancez, au moindre mouvement vous sautez. Armes bas.

Les brigands jettent les armes bas, et Catherine les tient en arrêt $»^{21}$.

Du discours à la mise en pratique, le dévouement républicain de l'héroïne se révèle sans faille et suscite une tirade élogieuse de l'époux dans l'avant-dernière scène qui achève de faire passer Catherine du rang d'anonyme à celui d'héroïne républicaine :

«SANS QUARTIER, à sa femme : Et toi, femme généreuse et sensible, viens recevoir, dans les bras de ton époux, au nom de la patrie, le nom d'héroïne quelle te donne, et que la postérité te conservera $\gg{ }^{22}$.

(20) Ibid., scène 9, p. 25.

(21) Ibid., scène 11, p. 27

(22) Ibid., scène 12, p. 29 
D'héroïne, la citoyenne de Saint-Milhier gardera surtout l'étiquette générique associée à sa ville natale plutôt que la postérité glorieuse. L'exemplarité de son action musclée et virile ne lui permit pas d'égaler un degré de célébrité équivalent à celui de Barra, référence tutélaire convoquée à maintes reprises dans les discours des personnages. Dans les deux premières scènes, Sans Quartier évoque avec son fils, La Bravoure, l'héroïsme du héros adolescent mort pour la patrie, donnant lieu à une remarque programmatique du père : «Eh bien! L'expérience nous prouve que les héros en France, sont de tout sexe et de tout âge [...] $»^{23}$. L'intrigue viendra en expliciter toute la portée. Que Barra constitue la seule référence héroïque des personnages prouve qu'un transfert générationnel s'est opéré : ces héroïnes de l'instant présent et de la génération révolutionnaire ne se réfèrent plus aux héros de l'Antiquité ou à des modèles aristocratiques. L'émulation se produit désormais au niveau horizontal entre les membres de la fratrie citoyenne et non d'après une hiérarchie verticale. Peut-être faut-il voir dans cette course effrénée à l'héroïsme, dépourvue de références culturelles autres que celles de la décennie révolutionnaire, l'une des raisons de leur absence de postérité au-delà de leur contexte d'émergence.

\section{Portrait de l'héroïne anonyme sous la Terreur : Liberté, famille, patrie}

La vogue des faits historiques connaît son apogée pendant la période qui va de l'an II à Thermidor, soutenue par une propagande massive à destination du peuple sans-culotte. La promotion de l'héroïne prend appui sur une représentation radicalement manichéenne : en bonne républicaine, l'héroïne doit sacrifier ce qu'elle a de plus cher à la patrie, situation qui donne lieu à une tension dramatique accentuée par la brièveté épurée du trait historique sur le plan de l'action. Conformément au souhait exprimé par les députés de la Convention en préambule de leur Recueil des actions héroïques, celles-ci ne sauraient se résumer au coup d'éclat lors d'un combat. La bravoure de l'héroïne de Mithier fait même exception dans le corpus. Un rapide repérage sur la nature de l'action héroïque représentée nous permet de mieux cerner ce que recouvre la notion d'héroïsme au féminin pour les hommes de l'an II : le dévouement à la patrie et la loyauté républicaine en constituent les principes fondamentaux. L'accès à la dignité héroïque de la figure féminine passe par la

(23) Ibid., scène 1, p. 4. 
mise en valeur de son rôle social en tant qu'épouse ou mère, premier vecteur d'intégration à la communauté républicaine. Dès lors, l'expression ardente de l'attachement à la patrie et de la force du sentiment patriotique suffit à légitimer le rôle politique de l'héroïne dans l'action. Cette représentation s'arc-boute sur la valorisation d'une société égalitaire au sein de laquelle la femme, matrone républicaine, permet la cohésion sociale par l'éducation et la transmission des valeurs républicaines. C'est sans doute la raison pour laquelle ce corpus abondant des traits historiques représentés autour de l'an II présente une portée idéologique qui se révèle moins stéréotypée qu'elle n'en a l'air. À l'opposé de la tentation d'associer la figure féminine à l'esprit de conservatisme politique, calqué sur la conception d'un rôle biologique de la femme, ces citoyennes républicaines prouvent qu'au prix d'un effort sur soi, il est possible d'être autant - voire davantage - héroïque qu'un homme.

Dans La Vraie républicaine ou la voix de la patrie ${ }^{24}$, comédie en un acte représentée au cours du mois de messidor an II, l'intrigue est centrée sur la question du mariage : une jeune veuve, ancienne aristocrate désormais convertie aux valeurs républicaines, la citoyenne d'Offémont, souhaite se remarier avec le citoyen Dumont, un jeune fermier qu'elle emploie. Le projet est aussitôt dénoncé par le frère de la citoyenne, nommé d'Appréville, ennemi de la Révolution, qui confie au prêtre Doucin le dégoût que lui inspire une telle mésalliance. Prêt à tout pour empêcher ce mariage - qui déshériterait au passage sa fille, unique héritière de la citoyenne -, d'Appréville n'hésite pas à comploter avec un général autrichien ennemi, sur le point d'attaquer la ville voisine : il soutiendra son offensive si le général promet en retour de faire échouer ce projet de mariage. Instruite que son frère a trahi la patrie, la citoyenne le dénonce au maire qui le fait immédiatement arrêter. Et la pièce de se conclure sur l'éloge en bonne et due forme de la vertu civique de la citoyenne par le maire :

«Citoyenne d'Offémont: tu viens de faire une action qui t'élève au rang des plus célèbres héroïnes. Tu t'es séparée d'une famille qui n'était pas digne de te posséder, et tu acquiers, pour frères, tous les Citoyens qui s'intéressent au salut de la République et à l'affermissement de la Liberté. Oublie à jamais d'Appréville; il est déjà effacé de notre mémoire $»^{25}$.

(24) Citoyen Laus, La Vraie Républicaine ou la voix de la patrie, comédie en un acte et en prose, représentée pour la première fois au Théâtre du Lycée des Arts au cours du mois de messidor an II, Paris, Impr. Cailleau, an II.

(25) Ibid., scène 13, p. 35-36. 
On célèbre ensuite le mariage républicain de la citoyenne et du fermier sur l'autel de la Patrie, en dansant la Carmagnole et en célébrant les vertus civiques de l'union patriotique. La dernière réplique de la pièce, prononcée par le maire, insiste sur le rôle social des citoyennes comme garantes de la pérennité des institutions républicaines : "Citoyennes, conservez toujours cet ardent patriotisme dans vos cœurs; faites-en partager la flamme à vos époux, à vos amans, à vos frères, et la République vous devra sa stabilité et sa gloire $»^{26}$. La mission républicaine conférée à l'épouse la fait triompher en championne active et positive du nouveau régime. Pour mettre en perspective cette représentation, il faudrait la comparer à l'ensemble du répertoire pro-jacobin, au sein duquel l'héroïne apparaît parfois sous un jour bien peu favorable, à l'image de l'épouse faussement convertie au républicanisme qui est représentée dans L'Epoux républicain de Pompigny ${ }^{27}$ : pétrie d'esprit contrerévolutionnaire, elle complote en réalité, aidée d'un chanoine, contre son époux patriote.

En renversant l'échelle axiologique des vertus traditionnellement associées à chaque sexe, les faits historiques de la période préthermidorienne placent l'héroïne dans une posture d'exemplarité morale. Dans La Discipline républicaine ${ }^{28}$, fait historique en un acte représenté en l'an II, la jeune républicaine Cécile, amante de Victor parti défendre la patrie contre les troupes ennemies de la République, revendique fièrement dès les premières scènes son caractère patriotique :

«JUSTINE : [...] tu sais que le devoir de Victor l'appelle au combat, et que...

CÉCILE : Eh! s'il balançait un moment à marcher contre les ennemis de la Patrie; si l'amour dérobait un seul instant à sa gloire, serait-il encore digne de moi? non! qu'il marche! qu'il vole! qu'il combatte! qu'il triomphe! et quand la République n'aura plus d'ennemis, alors la main de Cécile deviendra le prix de son dévouement, de son civisme et de sa valeur $»^{29}$.

(26) Ibid., scène 13, p. 39.

(27) Pompigny, L'Époux républicain, drame patriotique en deux actes et en prose, représenté pour la première fois sur le Théâtre de la Cité-Variétés, le 20 pluviôse an II (8 février 1794), Paris, Impr. Cailleau, 1794.

(28) Plancher de Valcour, La Discipline républicaine, fait historique en un acte et en prose représenté sur le Théâtre de l'opéra-comique national au mois de floréal an II, Paris, Impr. Cailleau, 1794.

(29) Ibid., scène 2, p. 6. 
$\mathrm{Au}$ cours de la même scène, la jeune héroïne repousse farouchement le stéréotype de l'amante tragique vouée à la déploration. Lorsque sa bonne amie évoque la perspective d'une mort prématurée de Victor lors d'un combat, elle répond avec appui :

«Eh! ma Justine, écartons ces pressentiments funestes. D’ailleurs, on ne calcule point avec la Patrie. Je sens que la perte de Victor m'arracherait au bonheur: mais qu'est-ce que le bonheur d'un individu, mis dans la balance avec le salut de la République? [...] Cécile donnerait des larmes à sa mort : mais elle aurait le courage de lui survivre. Elle aurait celui de donner sa main à un de ses compagnons d'armes. Ma mort serait inutile à la prospérité publique. L'esclave ne sait que mourir; l'être libre sait faire plus. En donnant des enfants à l'État, en les élevant dans l'amour de la Patrie, j'aurais rempli les devoirs d'une Républicaine, et l'ombre de son amant, s'il existe encore des souvenirs dans le tombeau, applaudirait à mon dévouement $»^{30}$.

L'expression d'une telle apologie du sacrifice, articulée à l'argument économique de l'utilité publique, paraît certainement caricaturale à notre sensibilité contemporaine. Il faut néanmoins la replacer dans son contexte de représentation marqué par le projet de régénération morale qui aspire à faire primer l'intérêt général et supérieur de la patrie sur l'intérêt particulier des citoyens. Ce discours à l'extrémisme rigoriste coïncide parfaitement avec la propagande familiale du gouvernement de salut public, non exempte de paradoxe : la femme y acquiert un rôle politique par l'exercice des vertus morales et civiques sans pourtant conquérir une véritable autonomie sociale qui la lie intrinsèquement à son rôle d'épouse et de mère.

Néanmoins, le répertoire des faits historiques a proposé dans certains cas une représentation alternative, à travers la promotion de l'hérö̈ne au militantisme viril, tout entière vouée à la lutte patriotique. La figure de la mère républicaine apparaît comme le personnage privilégié : en se révélant prête à sacrifier le lien filial à la fratrie métaphorique des citoyens, la mère républicaine atteint une forme d'héroïsme qui confine au sublime. Certes motivée par les directives de la propagande jacobine, la mise en valeur de cet héroïsme paradoxal sur les scènes théâtrales nous renseigne sur les schémas mentaux des contemporains. Le pôle du féminin se déplace de l'espace domestique où il était voué à la protection pour

(30) Ibid., scène 2, p. 6-7. 
investir l'espace de la place publique. On retrouve cette trajectoire symbolique dans le fait historique et patriotique intitulé Agricol Viala ou le jeune héros de la Durance ${ }^{31}$, représenté début juillet 1794 dans un contexte de radicalisation de la Terreur. Dans cette pièce, le jeune héros adolescent suit l'itinéraire du martyr républicain conformément aux récits des manuels héroïques. Mais la pièce se singularise par la présence de Pauline, mère du jeune Viala, qui se révèle au fur et à mesure de l'intrigue comme l'héroïne dramatique et idéologique de l'action. Lorsqu'à la deuxième scène, son fils lui témoigne la force de son attachement en lui déclarant : « Si j'avais deux cœurs, ils seraient tous les deux pour toi et pour toi seule », elle rappelle immédiatement l'intérêt suprême de la République par l'emploi de la métaphore familiale : «Et la République, mon cher Agricol? C'est bien la première mère, celle-là! $»^{32}$.

L'affirmation de sa citoyenneté passe par la mise à distance de tout ce qui pourrait la relier à un instinct biologique bassement féminin. Alors que son fils tente en permanence de solliciter la bienveillance maternelle, elle l'exhorte aux mâles vertus du combat: "Ce n'est que quitter une mère pour une autre, et celle-là doit passer avant tout; il faut abandonner tout le reste pour la défendre : car sans Patrie, il n'y a plus de famille $»^{33}$. Elle s'affirme d'autant mieux en chef charismatique de l'action que le jeune Agricol Viala apparaît naïf et indécis. Il se laisse berner par un des rebelles qui réussit à détourner son attention tandis que ses complices franchissent la Durance pour préparer l'assaut. Alors que les républicains, sur le point de lancer une contre-attaque défensive, hésitent à accepter le jeune Viala parmi leur troupe, sa mère l'exhorte à nouveau à prendre les armes pour sauver la patrie :

« Ne nous occupons que de la République, mon cher Agricol : voici ta véritable mère; et, si l'ennemi vient, ne songe qu'à elle; oublie ton âge; sois un homme! Il faut qu'aujourd'hui le laurier de la victoire ceigne ton front, ou que la palme civique couvre ton cercueil $»^{34}$.

Terrassé lors du combat, le jeune Viala s'effondre sous les yeux de sa mère et de ses frères d'armes. En fidèle et loyal fils de la patrie, il

(31) Citoyen Philipon, Agricol Viala ou le jeune héros de la Durance, fait historique et patriotique en un acte et en prose, représenté pour la première fois sur le Théâtre des Amis de la Patrie le 13 messidor an II ( $1^{\text {er }}$ juillet 1794), Paris, Barba, an II.

(32) Ibid., scène 2, p. 5.

(33) Ibid., scène 2, p. 6.

(34) Ibid., scène 6, p. 22. 
meurt en louant la République. D'abord frappé d'une douleur aux accents d'un noble pathétisme, la mère républicaine se ressaisit rapidement dans un élan d'altruisme républicain qui n'est pas sans rappeler la figure de Brutus qui avait suscité une vogue populaire dès 1791. La douleur du sacrifice filial est transcendée par la cause républicaine qui substitue au fils biologique tous les fils de la Patrie. La pièce se clôt ainsi sur la double exemplarité morale du héros martyr et de la mère républicaine :

«L'AIDE DE CAMP : Ne le pleurons pas. Mourir pour la patrie, c'est vivre pour la gloire. (à Pauline) Tendre et généreuse mère, songez que s'il vous est ravi, c'est dans le champ de l'honneur, et que le pays doit sa conservation au jeune héros qui vous dut le jour.

PAULINE : Mon fils n'est plus!... Qui me rendra ses tendres soins, ses caresses?

L'AIDE DE CAMP : Qui? Tous nos concitoyens. Un soldat qui meurt pour la République, laisse autant de fils à sa mère, qu'il reste de républicains. [...]

PAULINE, essuyant ses larmes, et prenant en quelque sorte un nouvel être : Ah! c'est vrai... il est mort pour la Patrie... je ne le pleure plus.

L'AIDE DE CAMP : Oui, c'est moins un tribut de larmes, qu'un tribut d'admiration, qui est dû à son dévouement généreux. Célébrons sa mémoire, et que les éloges donnés à sa vertu, engagent nos enfants à imiter son exemple (On emporte Agricol, et Pauline le suit) $»^{35}$.

Une ambition similaire anime Barra ou la mère républicaine ${ }^{36}$, drame historique en trois actes représenté en 1793. L'action et le discours y sont monopolisés par la mère du jeune héros, Dorothée, matrone républicaine face à laquelle le rejeton ne peut qu'apparaitre légèrement falot. La question de la dignité héroïque et du rôle politique des femmes au sein de la République est abordée en filigrane par la pièce dans l'opposition frontale de deux personnages féminins qui ne partagent pas la même ligne idéologique. La famille de Barra, qui a fui la Vendée pour échapper aux brigands contre-révolutionnaires, a trouvé refuge chez la citoyenne Brigitte : cette dernière exprime un discours modéré sur les violentes

(35) Ibid., scène 18, p. 27-28.

(36) Citoyenne Villiers, Barra ou la mère républicaine, drame historique en trois actes et en prose, représenté pour la première fois sur le théâtre de Dijon le 5 germinal an II (25 mars 1794), Dijon, Impr. P. Causse, an II. En dépit de sa caractérisation générique en drame historique, nous avons choisi d'intégrer la pièce à notre corpus car elle présente une dramaturgie et une esthétique semblables à celle des faits historiques. 
dérives engendrées par la Révolution, discours qui suscite l'indignation réprobatrice de Dorothée dans la tirade d'esprit pro-jacobin qui clôt la première scène de l'acte II :

« [...] Mais, quelles que soient ces peines, elles ne peuvent jamais donner le droit de déclamer contre la Révolution, et encore moins de s'opposer à son cours. Les révolutions des gouvernements, semblables aux grandes crises de la nature, bouleversent tout, pour tout régénérer. [...] Heureux! lorsque du sein du chaos où l'on est plongé, on peut voir, comme parmi nous, sortir l'ordre et la perfection! Et comment, et de quel front murmurer contre des événements d'où doit naitre la félicité publique? Mettre à part tout intérêt particulier, s'oublier soi-même, s'identifier entièrement au sort de la grande famille; voilà quelle est la vertu, voilà le patriotisme; et je ne reconnais pour vrais républicaines, que ceux qui sont capables de cet effort $»^{37}$.

Dans la scène finale, le récit de la mort héroïque du jeune Barra est à nouveau prétexte à un affrontement idéologique entre les deux femmes. L'intensité de la douleur exprimée par Dorothée touche à vif la sensibilité maternelle de Brigitte, qui exhorte les deux frères de Barra à modérer leurs ardeurs républicaines afin d'épargner à leur mère le déchirement d'une autre perte. Cette mise en garde suffit à raviver le fanatisme républicain de la mère éplorée :

«DOROTHÉE, se levant avec vivacité : Que dis-tu, Brigitte? quel faux zèle, quelle indiscrète amitié t'égare? Et pourquoi donc seraient-il nés? la patrie n'a-t-elle pas sur eux des droits plus sacrés que les miens? (à ses enfants avec beaucoup de véhémence) Mes enfants, vous, moi, nous sommes tous à elle; nos veilles, nos sueurs, notre sang; tout lui appartient. La plus grande gloire d'un Français est d'être appelé à l'honneur de la servir. O patrie! malheur à l'égoïste, dont l'insensible cœur n'est point embrasé de ton amour! mes enfants l'ont puisé dans mon sein : ils seront dignes de toi. O patrie! je te les dévoue. Leur frère n'est plus, il t'a payé sa dette. Et moi qui te le cédai, et moi qui lui survis... ah! que ne puis-je faire davantage? (La toile se baisse) $»^{38}$.

Au-delà des représentations idéologiques contrastées projetées sur la figure féminine, certains faits historiques proposent à travers leur

(37) Ibid., acte II, scène 1, p. 25-26.

(38) Ibid., acte III, scène 9 , p. 56. 
intrigue la mise en abyme d'un questionnement sur la nature héroïque des femmes. En mettant en scène une anecdote déjà bien connue des spectateurs populaires, la dramatisation opère moins au niveau des faits que dans la naissance d'une identité héroïque. C'est par exemple l'enjeu de l'intrigue d'un fait historique intitulé Les Chouans ou la républicaine de Malestroit $^{39}$, représenté en novembre 1794. Les citoyens Riou et Pain, auteurs de la pièce, placent d'emblée leur intrigue sous le paratexte tutélaire d'un extrait du Bulletin de la Convention du 6 vendémiaire rapportant l'anecdote historique suivante :

«La société populaire de Vannes instruit la Convention que les Brigands se sont portés dans la forêt de Trédion, dans la maison de Le Floch; qu'après avoir consommé toutes les subsistances qu'ils y trouvaient, ils forcèrent l'épouse de Le Floch de monter un de leurs chevaux, et d'aller à Malestroit s'informer si leurs camarades s'étaient emparés de cette ville, lui déclarant qu'ils retenaient en otage son mari et son enfant, qu'elle allaitait, pour la sûreté de ce voyage, qui devait être fait en six heures. Cette tendre mère arrose de larmes son mari et son enfants, les laisse au pouvoir des Brigands et se met en route; mais au lieu d'aller à Malestroit, elle se porte furtivement chez un bon citoyen du voisinage, qui avertit de suite les chefs de la force armée, et les Brigands sont obligés de fuir $»^{40}$.

En mettant en scène la nature héroïque de la citoyenne Floch, épouse du citoyen, dans une dynamique de révélation intérieure et sociale, les auteurs font ainsi triompher une nouvelle génération héroïque. Ce transfert symbolique est perceptible à travers la convocation au début de la pièce de l'anecdote du chevalier d'Assas qui, capturé par les ennemis lui intimant le silence, aurait fait preuve de résistance héroïque en lançant l'alerte à ses compagnons. L'évocation de cet exemple héroïque rapporté par le père de la Citoyenne Floch suscite un débat entre les deux époux sur la conduite à tenir en pareille situation :

«LA CITOYENNE FLOCH : J'aime ce trait sans l'admirer. Quel bon Français n'en ferait autant en pareille occasion? Je ne suis qu' une femme; mais certes j'aurais crié comme d'Assas : c'est l'ennemi!

(39) François-Marie-Joseph Riou et Joseph PAIN, Les Chouans ou la républicaine de Malestroit, trait historique en un acte et en prose, représenté pour la première fois à Brest le 16 brumaire an III (6 novembre 1794), Brest, Impr. Audran, 1794.

(40) Ibid., p. 2. 
LE CITOYEN FLOCH : D'accord, ma femme, tu aurais parlé; cela n'est pas douteux. Mais s'il eût fallu te taire pour sauver le régiment?

LA CITOYENNE FLOCH (un peu piquée) : Je l'aurais fait certainement. Juge de ce que je ferais pour sauver la patrie $»^{41}$.

La ferveur républicaine de la citoyenne Floch s'exprime par l'action comme lorsqu'après avoir trahi les brigands pour sauver la patrie, elle revient dans sa maison et libère son époux et son père gardés en otage par les officiers chouans. Son héroïsme viril suscite l'admiration collective et la postérité glorieuse de son action est déjà anticipée :

«L'OFFICIER: Républicains, dignes amis, quel tableau pour mes regards! quel charme pour mon cœur! Combien je suis heureux d'être l'instrument dont le ciel s'est servi pour votre délivrance, et pour la gloire de la république!... Mais nous devons tout... tout à cette excellente citoyenne. Elle a sauvé nos frères; elle a conduit mes pas; elle vous a rendus à la vie... Ah! toutes les couronnes civiques lui sont dues! je publierai son courage, ses vertus sublimes... La reconnaissance nationale va consacrer ce grand jour : les regards de la France entière vont se fixer sur ce modeste asile, et la postérité saura qu'une Française républicaine a tout à la fois bien mérité de la patrie, de la nature, de l'amour et de l'amitié $»^{42}$.

L'organisation simple et resserrée de l'intrigue autour de cet avènement du personnage féminin s'apparente à un réquisitoire visant à légitimer son nouveau statut héroïque : témoin de ce transfert générationnel, le père de la citoyenne loue son exploit en mentionnant que l'héroïsme d'Ancien Régime incarné par Assas est désormais surpassé par la gloire de l'héroïne populaire.

L'abondant corpus des faits historiques de la période jacobine confronte des représentations contrastées de l'héroïne qui excèdent les stéréotypes : en construisant le mythe de l'héroïne virile et vindicative au centre du système politique et social de la République, ces pièces proposent une réflexion sur la dignité hérö̈que des citoyennes et leur rôle actif au sein de la société comme vecteur d'intégration. Si la parenthèse de la Terreur tente d'établir la républicaine héroïque en pilier symbolique de la cohésion sociale, la période post-thermidorienne procède à un net infléchissement de la représentation.

(41) Ibid., scène 1, p. 11.

(42) Ibid., scène 13, p. 32-33. 


\section{La période post-thermidorienne : l'héroïne rend les armes et regagne le foyer.}

Après l'heure de gloire du fait historique et patriotique pendant la période de la dictature jacobine, la période qui va de Thermidor à la fin de la décennie révolutionnaire se caractérise par une désaffection progressive pour cette catégorie générique. À cette baisse numérique des faits historiques représentés s'ajoute un effet de dégénérescence dramatique qui rompt avec la tradition anecdotique pour s'orienter vers une esthétique mélodramatique en train de naître. La vocation politique et pédagogique des faits historiques ne disparaît pas mais elle se réoriente radicalement en changeant de cible : la chute du Grand Comité de l'an II permet l'émergence d'une critique politique et morale des dérives de la Terreur. La représentation de l'héroöne n'échappe pas à ce phénomène : l'héroïne post-thermidorienne est avant tout une victime du Comité de salut public.

On en trouve un bel exemple dans un fait historique intitulé Cange ou le commissionnaire bienfaisant ${ }^{43}$ représenté en octobre 1794, quelques mois après la chute de Robespierre. Ce fait historique se caractérise par la virulente condamnation morale du gouvernement de la Terreur dont Cange se plaît à prédire la chute prochaine. L'intrigue représente le combat de la citoyenne Georges, réduite à l'indigence, afin d'obtenir la libération de son époux incarcéré iniquement par le régime. À la scène 6 , l'héroïne est contrecarrée dans son projet par l'arrivée inopinée du citoyen Basset, caricature de l'ancien valet, jacobin parvenu se faisant désormais appeler Scaevola et jouissant d'une grande influence. Profitant de ce nouveau rapport de force, ce dernier propose à la citoyenne de plaider la cause de son époux à condition qu'elle consente à lui accorder ses faveurs au préalable. La citoyenne lui oppose un refus outré et ne craint pas de braver son pouvoir :

«BASSET : Quel langage! sais-tu bien, femme audacieuse! que j'ai mille moyens de me venger de ces étonnants propos?

LA CITOYENNE GEORGES : Je conviens qu'ils doivent te surprendre et que tu as rencontré peu de citoyens assez courageux pour te les tenir en face; mais apprends que cette terreur, par laquelle toi et tes

(43) Citoyens Viller et Gouffé, Cange ou le commissionnaire bienfaisant, trait historique en un acte représenté pour la première fois sur le Théâtre de la Cité-Variétés le 10 brumaire an III (31 octobre 1794), Paris, Impr. Plassan, s.d. 
complices nous ont comprimés à force de crimes, ne fera que retarder de quelques instants le supplice qui vous est dû, et qui effrayera à jamais ceux qui seraient tentés de suivre vos horribles traces $»^{44}$.

L'héroïsme du personnage féminin se manifeste ici dans l'expression de la vertu morale de l'épouse républicaine. À la fin de la pièce, le citoyen Georges est libéré grâce à l'intervention de Cange et salue l'exemplarité morale de son épouse, qui a su conserver sa dignité dans l'adversité. Néanmoins l'héroöne perd beaucoup en consistance dramatique : sa résistance idéologique se manifeste par ses discours et non ses actions. Elle déplore elle-même de ne pouvoir se rebeller efficacement. L'argument qu'elle convoque pour justifier son impuissance politique est significatif : elle rappelle son statut de mère qui l'oblige à faire preuve d'altruisme :

«Allez, monstres, si je n'étais qu'épouse, je défierais votre rage d'ajouter à mes tourments; mais je suis mère et ce titre doit me rendre sacrée à moi-même; je dois donc m'efforcer de lutter encore contre l'horreur de ma situation, et chercher quelques moyens de prolonger ma pénible existence et celle de ces infortunés... $»^{45}$.

En justifiant son renoncement à la posture héroïque, elle procède dans son discours au renversement total de l'idéologie du sacrifice familial prônée par la figure de la mère républicaine dans la période qui précède.

L'infléchissement idéologique de l'héroïne conduit au retour de la mise en valeur de son rôle conjugal et maternel, mais dans une perspective radicalement différente de celle des pièces des années 1793-1794. Les faits historiques post-thermidoriens procèdent à une réappropriation morale de la figure féminine du côté des valeurs traditionnelles d'éducation, de protection et de tendresse maternelles. Léonore ou l'amour conju$g a l^{46}$, fait historique représenté en l'an VI, remplit toutes les promesses de son titre, l'héroïne se dressant en parangon de piété et de dévouement conjugal sans limite. L'action est transposée en Espagne: Florestan, époux de Léonore, a été victime d'une incarcération abusive et est détenu

(44) Ibid., scène 6, p. 25.

(45) Ibid., scène 2, p. 7.

(46) Jean-Nicolas Boulluy, Léonore ou l'amour conjugal, fait historique en deux actes et en prose, représenté pour la première fois sur le Théâtre Feydeau, le $1^{\text {er }}$ ventôse an VI (19 février 1798), Paris, Barba, 1798. 
par le tyrannique et cruel Dom Pizare, gouverneur de la prison d'État. Résolue à agir par elle-même pour libérer son époux, Léonore décide de se travestir en homme - sous le nom de Fidelio - pour s'introduire dans le lieu carcéral où sa ruse fonctionne puisqu'elle est engagée en qualité de porte-clefs. Lors d'un passage chanté, elle exprime la force de son dévouement conjugal :

«Qu'il m'a fallu depuis deux ans

De courage et de patience!

Toujours sont des fardeaux pesants;

Nouveaux dangers, craintes, souffrances...

$\mathrm{Ah}$ ! je l'éprouve en ce moment,

Rien dans la nature n'égale

Ce feu sacré, ce sentiment

De la piété conjugale $»^{47}$.

Lorsque Dom Pizare décide d'assassiner de ses propres mains Florestan, elle se jette au-devant du corps de son époux pour le protéger du coup fatal, justifiant l'admiration de ce dernier : « Jamais ... non Jamais on ne poussa aussi loin l'héroïsme de l'amour $\rangle^{48}$. L'éloge du chœur final condense efficacement l'ambition morale de la pièce lorsque le ministre chante avec appui les vertus de l'épouse modèle :

«Vous qui, de Léonore, applaudissez le zèle,

La patience et l'intrépidité,

Femmes, prenez-la pour modèle,

Et faites consister, comme elle,

Votre bonheur dans la fidélité $»^{49}$.

Le retour à la sphère domestique et aux vertus familiales tend dès lors à amoindrir le rôle dramatique de l'héroïne reléguée dans certains cas à son statut de victime vouée à la déploration. C'est le cas dans Élize dans les bois ${ }^{50}$, comédie en un acte jouée en 1797 et que l'auteur présente comme la transposition dramatique d'un «fait historique du 14 thermidor », renforçant l'effet de contextualisation autour de la chute de Robespierre. L'héroïne y est décrite comme une victime des proscriptions,

(47) Ibid., acte I, scène 6, p. 18.

(48) Ibid., acte II, scène 4, p. 37.

(49) Ibid., acte II, scène 5, p. 40.

(50) SÉGUR LE Jeune, Élize dans les bois, fait historique du 14 thermidor, comédie en un acte et en prose, représenté à Paris sur le Théâtre de la Citoyenne Montansier, Paris, Huet, 1797. 
qui a vu périr ses proches sur l'échafaud et son mari se faire massacrer. Contrainte de prendre la fuite avec son fils, elle a trouvé refuge dans la forêt sous la protection du garde-bois Raimond, qui veille à sa survie et à sa sécurité. L'intrigue s'articule autour de la condamnation explicite de la Terreur et de ses excès. Cependant Élize n'y joue aucun rôle autre que celui de la déploration tragique. À plusieurs reprises sur le point de tomber dans la folie, elle apparaît comme une victime du renoncement, pour qui l'action politique n'est désormais plus envisageable.

Dans la période post-thermidorienne, la prévalence d'une forme d'héroïsme féminin plus domestique que politique sur les planches traduit-elle un retour conservateur au modèle patriarcal de la société prérévolutionnaire? La réponse mérite d'être nuancée. Sans doute cette nouvelle forme d'héroïsme au féminin se décline-t-elle à travers des personnages moins autonomes sur le plan dramatique et idéologique. Mais la politisation de la figure féminine se joue à un autre niveau, non plus dans l'émulation entre les sexes mais dans une refondation de la famille métaphorique des citoyens, au sein de laquelle les mères et les sœurs conservent leur égalité politique en tant que citoyennes. Le dilemme entre famille et patrie s'estompe au profit de la valorisation du rôle familial et social de la femme, certainement moins mythique que les exploits des citoyennes de l'an II, mais peut-être aussi plus fidèle à l'image de la femme sous la décennie révolutionnaire.

Que sont devenues les héroïnes de la Révolution? La très grande majorité a été oubliée, en dépit de la tentative de mythifier ces anonymes sorties de l'ombre pour défendre la patrie. Elles ont sans doute été victimes de leur succès et de la course à l'héroïsme encouragée par les autorités qui, dans la volonté politique de démocratiser les représentations idéologiques de l'héroïsme, a fini par tuer toutes les héroïnes à défaut d'en promouvoir une seule. Au-delà des bornes circonscrites de la décennie révolutionnaire, l'engouement populaire pour ces figures s'est éteint. Même la plus célèbre d'entre elles, l'héroïne de Mithier, n'aura pas de postérité au théâtre. L'approche par la catégorie générique du fait historique a permis de dégager quelques constantes dans la représentation; elle a surtout permis de mettre en évidence les processus dramatique et idéologique par lesquels s'opérait sur les scènes de théâtre la mythification de l'hérö̈ne populaire. Ce processus d'héroïsation a évolué au gré des bouleversements politiques et sociaux de la Révolution : sous le gouvernement de salut public, l'héroïne apparaît sous les traits d'une militante virile et androgyne qui conquiert une autonomie politique et sexuelle dans un 
esprit d'émulation perpétuel. L'influence de la propagande jacobine sur la représentation et la réception de ces héroïnes fut déterminante. Après Thermidor s'esquisse une évolution dans le répertoire : l'héroïne victime de la Terreur est valorisée dans son rôle familial et domestique; de manière symptomatique, son impact sur l'intrigue dramatique est amoindri. Faut-il interpréter ces éléments comme le retour à un conservatisme idéologique sonnant le glas de la mixité héroïque, ou comme le signe précurseur du rôle essentiellement familial dévolu à la femme lors la promulgation du Code civil en 1804? L'étude de ce répertoire nous montre qu'il faut se méfier des mythes politiques : le théâtre permet de mieux apprécier et réévaluer l'image des femmes pendant la décennie, de nuancer le préjugé rétrospectif de la misogynie de la société révolutionnaire. Malgré les débats enflammés des députés de la Convention sur la légitimité du choix d'une femme pour symboliser la République, c'est bien, après tout, la figure de Marianne que la postérité a choisi de retenir comme emblème républicain.

Eva Bellot

Université Sorbonne-Paris IV ANR THEREPSICORE

Centre d'Étude de la Langue et de la Littérature françaises des XVII et $\mathrm{XVIII}{ }^{\mathrm{e}}$ siècles evabellot@yahoo.fr 